\title{
Combining Many-Body Perturbation and Quantum Electrodynamics
}

\author{
Ingvar Lindgren, Sten Salomonson, and Daniel Hedendahl \\ Department of Physics, University of Gothenburg, 41296 Gothenburg, Sweden \\ Correspondence should be addressed to Ingvar Lindgren, ingvar.lindgren@physics.gu.se
}

Received 6 July 2011; Accepted 30 August 2011

Academic Editor: Alan Migdall

Copyright () 2011 Ingvar Lindgren et al. This is an open access article distributed under the Creative Commons Attribution License, which permits unrestricted use, distribution, and reproduction in any medium, provided the original work is properly cited.

It has been a long-sought problem to be able to combine many-body perturbation theory and quantum electrodynamics into a unified, covariant model. Such a model has recently been developed at our laboratory and is outlined in the present paper. The model has potential applications in many areas and opens up the possibility of studying the interplay between various interactions in different system. The model has so far been applied to highly ionized helium-like ions, and some numerical results are given. It is expected that the combined effect-that has never been calculated before-could have a significant effect on certain experimental data. The radiative effects are being regularized using the dimensional regularization in Coulomb gauge, and the first numerical results have been obtained.

\section{Introduction}

The many-body perturbation theory (MBPT) is now highly developed, and particularly all-order methods like the coupled-cluster approach (CCA) are very efficient and frequently used in atomic and molecular calculations $[1,2]$. There is nowadays a great research interest also in highly charged ions that can be effectively produced in modern high-energy accelerators. The purpose in this type of research is largely to study quantum electrodynamics (QED) in the presence of strong fields [3]. The experimental accuracy can here be quite high - in some cases far beyond the reach of any theoretical models presently available $[4,5]$. The shortcoming of these models is expected to be mainly due to the omission of the combined effect of QED and electron correlation.

The standard methods for MBPT can treat the electron correlation to essentially all orders of perturbation theory, but since they are noncovariant they cannot incorporate QED effects in any systematic way. Several covariant methods have been developed particularly for QED calculations on bound atomic systems, and most frequently used is the Smatrix formulation [6, Chapters 4-6]. These methods, however, have the disadvantage that they cannot be combined with MBPT, and they are for practical reasons limited to twophoton exchange. Particularly for lighter systems, this yields an incomplete treatment of the electron correlation.

It has been a long-sought problem to be able to calculate combined MBPT and QED effects in a systematic way, which could have important implications in various areas. The main obstacle, however, has been that the MBPT and QED procedures have quite different structures. (In 1988 a research programme at the Institute of Theoretical Physics in Santa Barbara, "Relativistic, Quantum electrodynamic, and Weak Interaction Effects in Atoms" was largely devoted to this problem [7].) One of the methods for QED calculations; however, namely, the Covariant-Evolution-Operator (CEO) method, recently developed by the Gothenburg group $[8,9]$, has a structure that is similar to that of MBPT, and it can therefore serve as a basis for a unified procedure. Such a procedure has been developed by the group during the last few years and is described in a number of publications $[10,11]$ as well as in a recent book [6]. The procedure is now being implemented [12-15], and at a later stage interesting comparison can be made with accurate experimental data that presently cannot be explained. The developments made so far are summarized in the present article. 


\section{Time-Independent Many-Body Perturbation Theory}

As an introduction we shall consider the basic many-body perturbation theory (MBPT), as described, for instance, in the book by Lindgren and Morrison, Atomic Many-Body Theory [1].

2.1. Bloch Equation. Many MBPT calculations in physics and quantum chemistry are based upon the generalized Bloch equation. We have then a set of target states satisfying the Schrödinger equation

$$
H\left|\Psi^{\alpha}\right\rangle=E^{\alpha}\left|\Psi^{\alpha}\right\rangle \quad(\alpha=1 \cdots d)
$$

and for each target state there exist model states, $\left|\Psi_{0}^{\alpha}\right\rangle(\alpha=$ $1 \ldots d)$, which are assumed to be linearly independent and form a model space. The projection operator for the model space is $P$, which together with the projection operator for the complementary space $Q$, forms the identity operator

$$
P+Q=I
$$

A single wave operator, $\Omega$, transforms all model states to the corresponding target states,

$$
\Omega\left|\Psi_{0}^{\alpha}\right\rangle=\left|\Psi^{\alpha}\right\rangle \quad(\alpha=1 \cdots d) .
$$

An effective Hamiltonian, operating in the model space, generates the exact energy when operating on the model state

$$
H_{\mathrm{eff}}\left|\Psi_{0}^{\alpha}\right\rangle=E^{\alpha}\left|\Psi_{0}^{\alpha}\right\rangle \quad(\alpha=1 \cdots d) .
$$

This leads to

$$
\Omega H_{\text {eff }}\left|\Psi_{0}^{\alpha}\right\rangle=E^{\alpha}\left|\Psi^{\alpha}\right\rangle
$$

which according to the Schrödinger equation (1) is equal to $H \Omega\left|\Psi_{0}^{\alpha}\right\rangle$. These relations hold for the entire model space, leading to the generalized Bloch equation

$$
\Omega H_{\mathrm{eff}} P=H \Omega P .
$$

In intermediate normalization (IN)

$$
\left\langle\Psi_{0}^{\alpha} \mid \Psi^{\alpha}\right\rangle=1 ; \quad\left|\Psi_{0}^{\alpha}\right\rangle=P\left|\Psi^{\alpha}\right\rangle \quad(\alpha=1 \cdots d),
$$

the effective Hamiltonian (4) becomes

$$
H_{\text {eff }}=P H \Omega P \text {. }
$$

The Hamiltonian is normally partitioned into

$$
H=H_{0}+V
$$

where $\mathrm{H}_{0}$ a model Hamiltonian, containing the sum of singleelectron Hamiltonians, and $V$ is a perturbation. This leads to the commonly used form of the generalized Bloch equation $[1,16]$

$$
\left[\Omega, H_{0}\right] P=Q\left(V \Omega-\Omega V_{\text {eff }}\right) P,
$$

where

$$
V_{\text {eff }}=H_{\text {eff }}-P H_{0} P=P V \Omega P
$$

is the effective interaction.

In the graphical representation the wave operator is under general conditions (complete model space, see [1]) represented by linked diagrams, which is the linked-diagram theorem, first demonstrated for closed-shell systems by Brueckner [17] and Goldstone [18] and later generalized by Brandow [19] and Lindgren [16] to general open-shell systems. This can be expressed

$$
\left[\Omega, H_{0}\right] P=Q\left(V \Omega-\Omega V_{\text {eff }}\right)_{\text {linked }} P
$$

a form of the generalized Bloch equation frequently used as the starting point for many-body perturbation calculations in physics and quantum chemistry. It is valid also for a manydimensional model space that does not have to be degenerate. The last term is referred to as the folded part, since it is conventionally represented graphically by folded diagrams [19] (see Figure 1).

2.2. All-Order Procedures. The Bloch equation can be iterated, leading to all-order procedures, as illustrated by the pair function (see Figure 2)

$$
\left|\rho_{a b}\right\rangle=\Gamma_{Q}\left(E_{0}\right) I^{\text {Pair }}|a b\rangle=: \Omega_{\mathrm{I}}|a b\rangle .
$$

Here

$$
\Gamma\left(E_{0}\right)=\frac{1}{E_{0}-H_{0}} ; \quad \Gamma_{Q}\left(E_{0}\right)=Q \Gamma\left(E_{0}\right)
$$

are the resolvent and reduced resolvent, respectively, and $E_{0}$ the energy of the initial state.

The all-order pair function satisfies a Dyson-type equation

$$
\begin{aligned}
\Gamma_{Q}(\mathcal{E}) I^{\text {Pair }} P_{\mathcal{E}}=\Gamma_{Q}(\mathcal{E})( & +V \Gamma_{Q}(\mathcal{E}) I^{\text {Pair }} \\
& \left.-\Gamma_{Q}\left(\mathcal{E}^{\prime}\right) I^{\text {Pair }} P_{\mathcal{E}^{\prime}} I^{\text {Pair }} P_{\mathcal{E}}\right) P_{\mathcal{E}},
\end{aligned}
$$

where $V$ is the Coulomb interaction. This is represented graphically in Figure 3. The last term represents the folded contribution with a double resolvent. $P_{\mathcal{E}}, P_{\mathcal{E}^{\prime}}, \ldots$ are projection operators for parts of the model space with energies $\mathscr{E}, \mathcal{E}^{\prime}, \ldots$, respectively.

The pair equation (13) represents the important pair-correlation to all orders and is frequently used in calculations on atomic and molecular systems. An even more powerful technique is the coupled-cluster approach (CCA), where the wave operator is expressed in exponential form

$$
\Omega=\mathrm{e}^{S}=1+S+\frac{1}{2} S^{2}+\frac{1}{3 !} S^{3}+\cdots
$$

This has the effect, for instance, that including pair correlation into the cluster operator, yields the most important quadruple effects on the wave operator. This technique has 


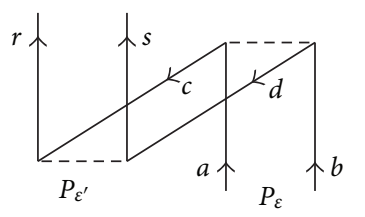

(a)

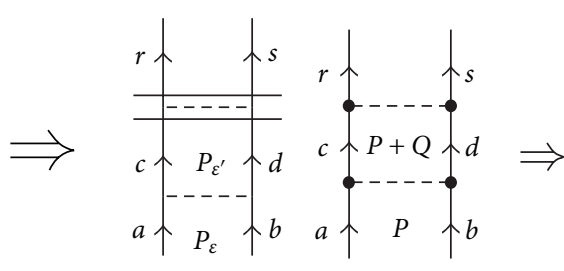$$
P_{\varepsilon} \uparrow
$$$$
\uparrow b
$$

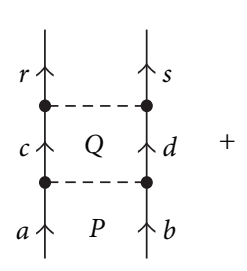

(b)

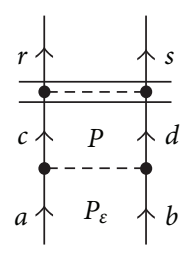

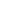

FIgURE 1: (a) Folded diagrams appear in the standard (Goldstone) graphical formulation of MBPT and represent the finite remainder after eliminating singularities due to intermediate model-space state, $|c d\rangle$. In a relativistic treatment, Feynman diagrams are normally used, and then the corresponding diagrams will be drawn straight (right). (b) The second-order diagram is separated into a regular part with the intermediate state in the model space and a remainder, due to the intermediate model-space state, represented by the folded diagram.

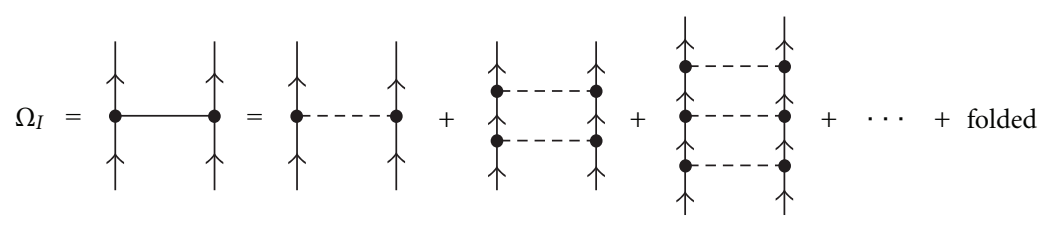

Figure 2: Graphical representation of the pair function (13).

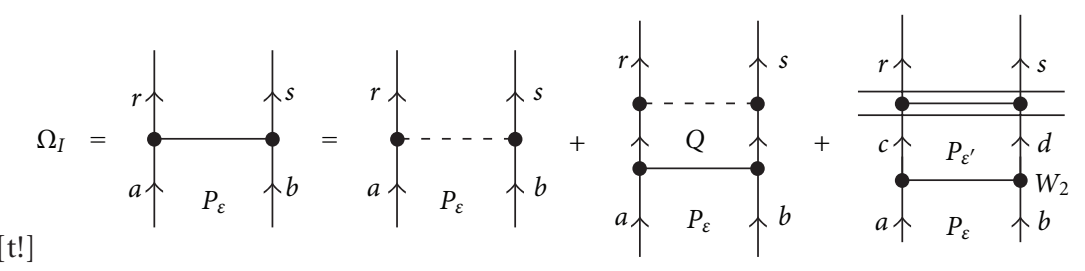

Figure 3: Graphical representation of the all-order pair equation (15). The last diagram represents the "folded" term. The double line represents the double denominator (double resolvent).

been extensively used during the last decades, particularly in quantum chemistry. For a review the reader is in particular referred to a recent review [2].

For open-shell systems it is convenient to apply the normal-ordered exponential Ansatz [20]

$$
\Omega=\left\{\mathrm{e}^{S}\right\}=1+S+\frac{1}{2}\left\{S^{2}\right\}+\frac{1}{3 !}\left\{S^{3}\right\}+\cdots,
$$

where the curly brackets represent normal ordering. In this way spurious contractions between open-shell operators are avoided.

In the CCA all diagrams are "connected" (for a complete model space), and the expansion can be expressed in analogy with (12) as (For the exact definitions of "linked" and "connected" diagrams, see, for instance, [1].)

$$
\left[S, H_{0}\right] P=Q\left(V \Omega-\Omega V_{\text {eff }}\right)_{\text {conn }} P .
$$

2.3. Relativistic MBPT. Relativistic MBPT is normally based upon the projected Dirac-Coulomb-Breit Hamiltonian [21]

$$
H_{\text {DCproj }}=\Lambda_{+}\left[\sum_{i=1}^{N} h_{D}(i)+V_{\mathrm{C}}+V_{\mathrm{B}}\right] \Lambda_{+},
$$

where the three terms represent the sum of single-particle Dirac Hamiltonians, Coulomb, and instantaneous Breit in- teractions between the electrons, respectively. The projection operators, $\Lambda$, are inserted in order to avoid negative-energy states that can lead to singularities (Breit-Ravenhall disease [22]). This approximation includes all effect up to order $\alpha^{2}$ Rydbergs (or $\alpha^{4} m c^{2}$ ), where $\alpha$ is the fine-structure constant. Effects beyond this approximation-of order $\alpha^{3}$ Rydbergs and higher-are conventionally referred to as QED effects (see further below).

\section{Covariant-Evolution Operator}

Mainly three methods for QED calculations on bound states have been developed (see [9, Section 5], [6, Chapter 6]). The standard procedure is the well-known S-matrix formulation, and more recently two other methods have been developed, the two-times Green's function technique, developed by the St Petersburg group [23], and the covariant-evolutionoperator (CEO) method, developed by the Gothenburg group [9]. The CEO method is particularly suitable for combining with MBPT and will be briefly summarized below.

3.1. Definition. The standard time-evolution operator, $U\left(t, t_{0}\right)$, describes in the interaction picture the evolution of the nonrelativistic time-dependent state vector

$$
|\chi(t)\rangle=N U\left(t, t_{0}\right)\left|\chi\left(t_{0}\right)\right\rangle \quad\left(t>t_{0}\right),
$$


where $N$ is a normalization constant. It should be noted that the evolution operator generally does not preserve the normalization, and in addition it can contain singularities. Therefore this equation should be handled with care.

The operator can be expanded (Relativistic units are used: $c=m=\hbar=\epsilon_{0}=1$.)

$$
\begin{gathered}
U\left(t, t_{0}\right)=\sum_{n=0}^{\infty} \frac{(-\mathrm{i})^{n}}{n !} \int_{t_{0}}^{t} \mathrm{~d} x_{1}^{4} \cdots \int_{t_{0}}^{t} \mathrm{~d} x_{n}^{4} T\left[\mathcal{H}\left(x_{1}\right) \cdots \mathcal{H}\left(x_{n}\right)\right] \\
\times \mathrm{e}^{-\gamma\left(\left|t_{1}\right|+\left|t_{2}\right| \cdots\right)}
\end{gathered}
$$

where an adiabatic damping is inserted in order to handle the singularities. The damping factor, $\gamma$, eventually goes to zero. $T$ is the Wick-time-ordering operator and $\mathscr{H}(x)$ is the perturbation density

$$
\mathscr{H}(x)=-\hat{\psi}^{\dagger}(x) e \alpha^{\mu} A_{\mu}(x) \hat{\psi}(x)
$$

corresponding to the time-dependent perturbation

$$
V(t)=\int \mathrm{d}^{3} \mathbf{x} \mathscr{H}(t, \mathbf{x})
$$

We note that this represents the emission/absorption of a single photon and, hence, operates in the extended Fock space with a variable number of photons. Two such interactions are needed to form the exchange of a virtual photon between the electrons (see Figure 4).

The field-theoretical Green's function can in the closedshell case be defined

$$
G\left(x, x_{0}\right)=\frac{\left\langle 0_{\mathrm{H}}\left|T\left[\widehat{\psi}_{\mathrm{H}}(x) \hat{\psi}_{\mathrm{H}}^{\dagger}\left(x_{0}\right)\right]\right| 0_{\mathrm{H}}\right\rangle}{\left\langle 0_{\mathrm{H}} \mid 0_{\mathrm{H}}\right\rangle}
$$

where $\hat{\psi}_{\mathrm{H}}, \hat{\psi}_{\mathrm{H}}^{\dagger}$ are the electron-field operators in the Heisenberg representation. The state $\left|0_{\mathrm{H}}\right\rangle$ is the "vacuum in the Heisenberg representation," that is, the state in the Heisenberg representation with no particles or holes. The numerator is

$$
\left\langle 0_{\mathrm{H}} \mid 0_{\mathrm{H}}\right\rangle=\langle 0|S| 0\rangle,
$$

where $\langle 0|$ is the vacuum state in the interaction picture and $S=U(\infty,-\infty)$ is the S-matrix. This leads to the expansion

$$
\begin{aligned}
G\left(x, x_{0}\right)= & \frac{1}{\langle 0|S| 0\rangle} \sum_{n=0}^{\infty} \frac{(-\mathrm{i})^{n}}{n !} \int \mathrm{d}^{4} x_{1} \cdots \int \mathrm{d}^{4} x_{n} \\
& \times\left\langle 0\left|T\left[\hat{\psi}(x) \mathscr{H}\left(x_{1}\right) \cdots \mathscr{H}\left(x_{n}\right) \hat{\psi}^{\dagger}\left(x_{0}\right)\right]\right| 0\right\rangle \\
& \times \mathrm{e}^{-\gamma\left(\left|t_{1}\right|+\left|t_{2}\right| \cdots\right)} .
\end{aligned}
$$

The standard evolution operator (20) operates only in the forward direction and is graphically represented by diagrams with outgoing particle lines (of positive energy). The operator for single-photon exchange between the electrons is illustrated in the first diagram in Figure 5. This operator is not covariant and consequently cannot be used in a relativistic
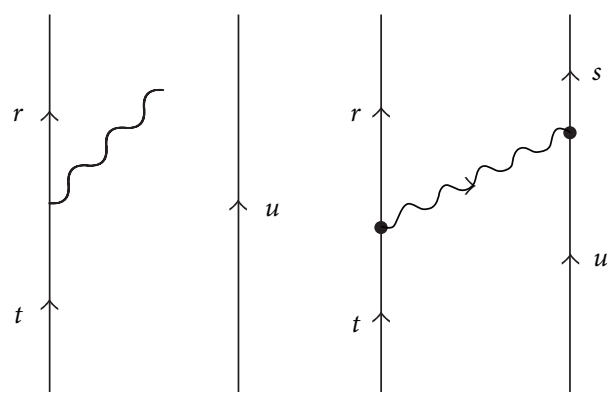

FIGURE 4: The single-photon exchange between the electrons is in the covariant-evolution operator represented by two perturbations (23).

treatment. Green's function (24), on the other hand, with electron propagators on the free ends is covariant (second diagram). By attaching free-electron lines to the graphical representation of Green's function, we will represent an evolution operator that is covariant, the covariant-evolution operator (CEO; third diagram).

The covariant-evolution operator is the evolution operator for the relativistic state vector in analogy with (20)

$$
\left|\chi_{\mathrm{Rel}}^{\alpha}(t)\right\rangle=N^{\alpha} U_{\mathrm{Cov}}\left(t, t_{0}\right)\left|\chi_{\mathrm{Rel}}^{\alpha}\left(t_{0}\right)\right\rangle
$$

which also generally lies in the extended space (in the following we shall drop the subscripts "Cov" and "Rel").

The single-particle covariant-evolution operator can generally be expressed

$$
\begin{aligned}
& U^{1}\left(t, t_{0}\right)=\iint \mathrm{d}^{3} \mathbf{x} \mathrm{d}^{3} \mathbf{x}_{0} \hat{\psi}^{\dagger}(x)\left\langle 0_{\mathrm{H}}\left|T\left[\hat{\psi}_{\mathrm{H}}(x) \hat{\psi}_{\mathrm{H}}^{\dagger}\left(x_{0}\right)\right]\right| 0_{\mathrm{H}}\right\rangle \\
& \times \hat{\psi}\left(x_{0}\right) \mathrm{e}^{-\gamma\left(\left|t_{1}\right|+\left|t_{2}\right| \cdots\right)}
\end{aligned}
$$

involving the same vacuum expectation as in the definition of the single-particle Green's function (24), apart from the fact that we shall here allow for uncontracted photons.

The covariant-evolution operator for single-photon exchange, operating on the unperturbed state, $|a b\rangle$, of energy $E_{0}$ becomes (leaving out the damping factor)

$$
U_{\mathrm{sp}}(t,-\infty)|a b\rangle=\mathrm{e}^{-\mathrm{i} t\left(E_{0}-H_{0}\right)} \Gamma\left(E_{0}\right) V_{\mathrm{sp}}|a b\rangle,
$$

where $\Gamma\left(E_{0}\right)$ is the resolvent (14). When only positive-energy states are involved (no virtual pairs), the potential $V_{\mathrm{sp}}$ is given by

$$
\begin{aligned}
&\left\langle r s\left|V_{\mathrm{sp}}\right| a b\right\rangle \\
&=\langle r s| \int_{0}^{\infty} \mathrm{d} k f(\kappa)\left[\frac{1}{\varepsilon_{a}-\varepsilon_{r}-(\kappa-\mathrm{i} \gamma)}\right. \\
&\left.+\frac{1}{\varepsilon_{b}-\varepsilon_{s}-(\kappa-\mathrm{i} \gamma)}\right]|a b\rangle,
\end{aligned}
$$

where $f(\kappa)$ is a gauge-dependent function of the magnitude of the photon momentum, $\kappa=|\mathbf{k}|$. 


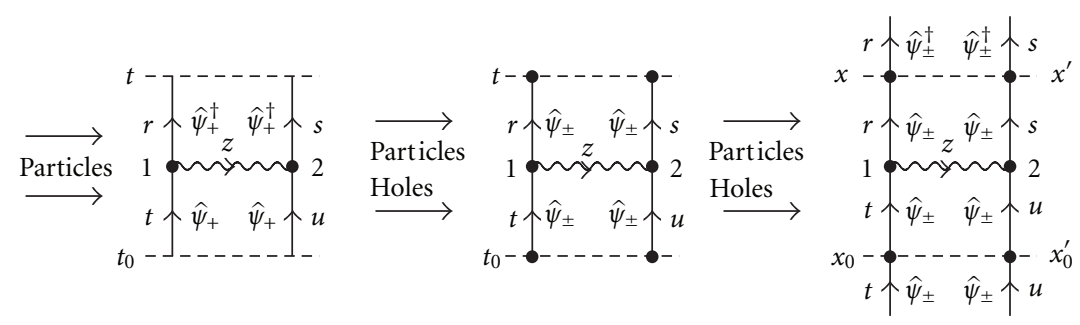

FIGURE 5: Comparison between the standard evolution operator, Green's function, and the covariant-evolution operator for single-photon exchange in the equal-time approximation [6, Figure 8.1].

In contrast to the situation in the S-matrix formulation, the initial and final states can in the covariant-evolutionoperator formalism have different energies. When the initial and final states do have the same energy, the potential (30) above becomes

$$
\left\langle c d\left|V_{\mathrm{sp}}\right| a b\right\rangle=\left\langle c d\left|\int_{0}^{\infty} \frac{2 \kappa \mathrm{d} \kappa f(\kappa)}{q^{2}-\kappa^{2}+\mathrm{i} \gamma}\right| a b\right\rangle
$$

$\left(q=\varepsilon_{a}-\varepsilon_{c}=\varepsilon_{d}-\varepsilon_{b}\right)$ which is the energy-conservative Smatrix result.

In a ladder of single-photon-exchange, involving only positive-energy states and interacting to the far right on the unperturbed state of energy $E_{0}$, the general potential becomes (see Figure 6)

$$
\begin{aligned}
\left\langle r s\left|V_{\text {sp }}\left(E_{0}\right)\right| t u\right\rangle & \\
=\langle r s| \int_{0}^{\infty} \mathrm{d} k f(\kappa)[ & \frac{1}{E_{0}-\varepsilon_{r}-\varepsilon_{u}-(\kappa-\mathrm{i} \gamma)} \\
& \left.+\frac{1}{E_{0}-\varepsilon_{t}-\varepsilon_{s}-(\kappa-\mathrm{i} \gamma)}\right]|t u\rangle .
\end{aligned}
$$

If there are no model-space states involved, the covariantevolution operator for the complete ladder becomes

$$
\begin{aligned}
U_{0}(t,-\infty)_{\mathrm{Ladd}} P= & \mathrm{e}^{-\mathrm{i} t\left(E_{0}-H_{0}\right)} \Gamma_{Q}\left(E_{0}\right) V_{\mathrm{sp}}\left(E_{0}\right) \\
& \times \Gamma_{Q}\left(E_{0}\right) V_{\mathrm{sp}}\left(E_{0}\right) \cdots \Gamma_{Q}\left(E_{0}\right) V_{\mathrm{sp}}\left(E_{0}\right) P .
\end{aligned}
$$

We note that the energy parameter of all potentials and resolvents as well as the time factor is the energy of the unperturbed state to the far right.

3.2. Green's Operator. The covariant-evolution operator (CEO) becomes singular (or quasisingular) when a state degenerate (or quasidegenerate) with the initial state is involved. In the definition of Green's function in the closed-shell case (24) the corresponding singularities are eliminated by dividing by the expectation value of the S-matrix. In the CEO case a different procedure is needed.

We define the Green's operator in the general Fock space by the relation

$$
U\left(t, t_{0}\right) P=g\left(t, t_{0}\right) \cdot P U\left(0, t_{0}\right) P
$$

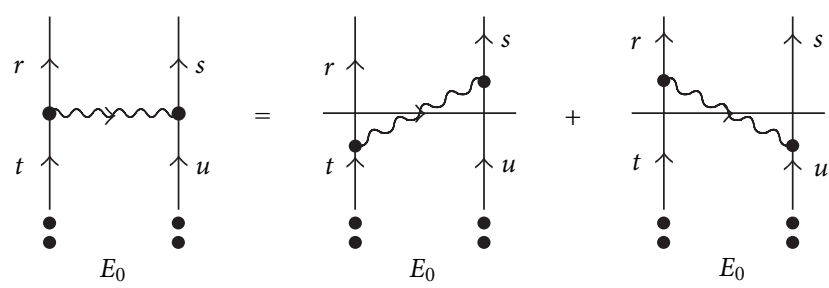

FIgURE 6: The evolution-operator diagram for single-photon exchange.

where the operator to the left of the heavy dot does not operate beyond the dot. This implies that the energy parameter of Green's operator above is that of the intermediate modelspace state. The definition leads to the expansion (with $t_{0}=$ $-\infty)$

$$
\begin{aligned}
g^{(0)}(t, \mathcal{E}) P_{\mathcal{E}}= & U^{(0)}(t, \mathcal{E}) P_{\mathcal{E}} \\
g^{(1)}(t, \mathcal{E}) P_{\mathcal{E}}= & U^{(1)}(t, \mathcal{E}) P_{\mathcal{E}}-g^{(0)}\left(t, \mathcal{E}^{\prime}\right) \cdot P_{\mathcal{E}^{\prime}} U^{(1)}(0, \mathcal{E}) P_{\mathcal{E}} \\
g^{(2)}(t, \mathcal{E}) P_{\mathcal{E}}= & U^{(2)}(t, \mathcal{E}) P_{\mathcal{E}}-g^{(0)}\left(t, \mathcal{E}^{\prime \prime}\right) \cdot P_{\mathcal{E}^{\prime \prime}} U^{(2)}(0, \mathcal{E}) P_{\mathcal{E}} \\
& -g^{(1)}\left(t, \mathcal{E}^{\prime}\right) \cdot P_{\mathcal{E}^{\prime}} U^{(1)}(0, \mathcal{E}) P_{\mathcal{E}}
\end{aligned}
$$

and so forth.

As before, $P_{\varepsilon}, P_{\varepsilon^{\prime}}, \ldots$ are projection operators for the parts of the model space with the energies $\mathscr{E}, \mathscr{E}^{\prime}, \ldots$, and the relevant energy parameters of the operators are indicated. The negative "counterterms" cancel the singularities, making the Green's operator regular. We note that with the perturbation (22) the term $P_{\mathcal{E}^{\prime}} U^{(1)}(0, \mathcal{E}) P_{\mathcal{E}}$ with only a single uncontracted photon vanishes.

It follows from the definition (27) that

$$
\left|\chi^{\alpha}(t)\right\rangle=N^{\alpha} U(t,-\infty)\left|\Phi^{\alpha}\right\rangle
$$

where

$$
\left|\Phi^{\alpha}\right\rangle=\lim _{t-\infty}\left|\chi^{\alpha}(t)\right\rangle
$$

is the "parent state." For the time $t=0$ we have

$$
\left|\chi^{\alpha}(0)\right\rangle=\left|\Psi^{\alpha}\right\rangle=N^{\alpha} U(0,-\infty)\left|\Phi^{\alpha}\right\rangle .
$$


The target state $\left|\Psi^{\alpha}\right\rangle$ is intermediately normalized (7) if

$$
N^{\alpha}=\left\langle\Psi_{0}^{\alpha}|U(0,-\infty)| \Phi^{\alpha}\right\rangle^{-1},
$$

where $\left|\Psi_{0}^{\alpha}\right\rangle$ is the corresponding model state. This leads to

$$
\left|\chi^{\alpha}(0)\right\rangle=\left|\Psi^{\alpha}\right\rangle=\lim _{\gamma \rightarrow 0} \frac{U(0,-\infty)\left|\Phi^{\alpha}\right\rangle}{\left\langle\Psi_{0}^{\alpha}|U(0,-\infty)| \Phi^{\alpha}\right\rangle}
$$

which is a generalization of the Gell-Mann and Low theorem [24], valid also for a multidimensional model space [9]. Here, both the numerator and denominator are normally singular, but the ratio is regular in the limit when $\gamma \rightarrow 0$.

Using the definition of Green's operator (34), we have from (36)

$$
\left|\chi^{\alpha}(t)\right\rangle=N^{\alpha} g(t,-\infty) \cdot P U(0,-\infty)\left|\Phi^{\alpha}\right\rangle=g(t,-\infty)\left|\Psi_{0}^{\alpha}\right\rangle,
$$

since

$$
N^{\alpha} P U(0,-\infty)\left|\Phi^{\alpha}\right\rangle=P\left|\Psi^{\alpha}\right\rangle=\left|\Psi_{0}^{\alpha}\right\rangle
$$

is the model state. This implies that Green's operator acts as a wave operator for the relativistic state vector at all times. In particular, the covariant analogue of the MBPT wave operator (3) becomes

$$
\Omega=\mathcal{G}(0,-\infty)
$$

which is also a Fock-space operator. This gives the connection between the CEO formalism and standard MBPT.

3.3. The Many-Body Hamiltonian. (See [6, Sections 6.4, 5].) The state vector $(40),\left|\Psi^{\alpha}\right\rangle$, satisfies a relativistic "Schrödinger-like" eigenvalue equation

$$
H\left|\Psi^{\alpha}\right\rangle=\left(H_{0}+V_{\mathrm{F}}\right)\left|\Psi^{\alpha}\right\rangle=E^{\alpha}\left|\Psi^{\alpha}\right\rangle \text {. }
$$

When the Coulomb gauge is used (as we shall normally assume to be the case here), the perturbation, $V_{\mathrm{F}}$, is given by the Coulomb interaction, $V_{\mathrm{C}}$, and the transverse part of the perturbation (23). The total many-body Hamiltonian then becomes in second quantization

$$
\begin{aligned}
H= & \int \mathrm{d}^{3} \mathbf{x} \hat{\psi}^{\dagger}(x)\left(\boldsymbol{\alpha} \cdot \hat{\mathbf{p}}+\beta m+v_{\text {ext }}(x)-e \alpha^{\mu} A_{\mu}(x)\right) \hat{\psi}(x) \\
& +H_{\mathrm{Rad}}+\frac{1}{2} \iint \mathrm{d}^{3} \mathbf{x}_{1} \mathrm{~d}^{3} \mathbf{x}_{2} \hat{\psi}^{\dagger}\left(x_{1}\right) \hat{\psi}^{\dagger}\left(x_{2}\right) V_{\mathrm{C}} \hat{\psi}\left(x_{2}\right) \hat{\psi}\left(x_{1}\right),
\end{aligned}
$$

where $v_{\text {ext }}(x)$ is the external (usually nuclear) potential. This is Fock-space operator, operating in the extended space with unpaired photons. Since the number of photons is not conserved, also the radiation-field Hamiltonian, $H_{\mathrm{Rad}}$, is included.

Using Fock-space operators, the effective interaction (11) becomes

$$
V_{\text {Feff }}=P V_{\mathrm{F}} \Omega P
$$

The effective interaction still operates in the model space, which is a part of the restricted space with no unpaired photons.

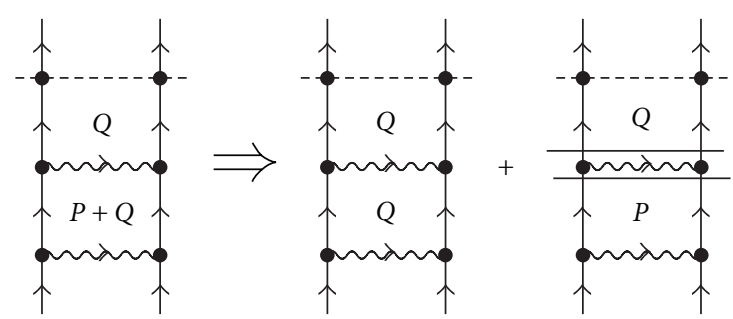

FIgURE 7: Elimination of the singularity of the second-order evolution operator due to an intermediate model-space state (49) (c.f. Figure 1).

3.4. Model-Space Contribution. (See [10, 11], [6, Section 6.7].) The second-order Green's-operator expression (35) is for $t=0$

$$
\mathcal{g}^{(2)}(0, \mathcal{E}) P_{\mathcal{E}}=Q U^{(2)}(0, \mathcal{E}) P_{\mathcal{E}}-\mathcal{g}^{(1)}\left(0, \mathcal{E}^{\prime}\right) \cdot P_{\mathcal{E}^{\prime}} U^{(1)}(0, \mathcal{E}) P_{\mathcal{E}}
$$

using the fact that $G^{(0)}(0)=1$. We assume here that the perturbation is the complete photon exchange (32). The secondorder CEO then becomes

$$
U^{(2)}(0, \mathcal{E}) P_{\mathcal{E}}=\Gamma(\mathcal{E}) V(\mathcal{E})(P+Q) \Gamma(\mathcal{E}) V(\mathcal{E}) P_{\mathcal{E}} .
$$

This will be (quasi)singular, if the intermediate state lies in the model space. The singularity is cancelled by the counterterm, leaving a finite contribution,

$$
\begin{aligned}
& \mathscr{g}^{(2)}(0, \mathcal{E}) P_{\mathcal{E}} \\
& \quad=\Gamma_{Q}(\mathscr{E}) V(\mathscr{E}) \Gamma_{Q}(\mathscr{E}) V(\mathcal{E}) P_{\mathcal{E}}+\frac{\delta \mathcal{g}^{(1)}(0, \mathcal{E})}{\delta \mathcal{E}} P_{\mathcal{E}^{\prime}} V(\mathcal{E}) P_{\mathcal{E}},
\end{aligned}
$$

where $\delta g^{(1)}(0, \mathcal{E})=g^{(1)}(0, \mathcal{E})-\mathcal{g}^{(1)}\left(0, \mathcal{E}^{\prime}\right)$ and $\delta \mathcal{E}=\mathcal{E}-$ $\mathcal{E}^{\prime}$. This is illustrated in Figure 7. In the case of complete degeneracy the difference ratio turns into a partial derivative. The finite contribution is referred to as the model-space contribution (MSC). If the interaction is energy independent, this becomes identical to the corresponding folded contribution in standard MBPT (Figure 1).

Generalizing the procedure above to arbitrary orders, leads to [10, Equation (100)], [11, Equation (64)], [6, Equation (6.96)]

$$
g(0, \mathcal{E}) P_{\mathcal{E}}=g_{0}(0, \mathcal{E}) P_{\mathcal{E}}+\sum_{n=1} \frac{\delta^{n} \mathcal{g}_{0}(0, \mathcal{E})}{\delta \mathcal{E}^{n}}\left(V_{\mathrm{eff}}\right)^{n} P_{\mathcal{E}}
$$

where $V_{\text {eff }}$ is the effective interaction (11). The first term above represents Green's operator without any folds

$g_{0}(0, \mathcal{E})=1+\Gamma_{Q}(\mathcal{E}) V(\mathcal{E})+\Gamma_{Q}(\mathcal{E}) V(\mathscr{E}) \Gamma_{Q}(\mathcal{E}) V(\mathcal{E})+\cdots$

Consequently the last term of (50) represents the effects of all folds. 
Operating with (50) on the model function in the case of a one-dimensional model space (single reference) of energy $E_{0}$, gives

$$
\mathcal{g}\left(0, E_{0}\right)\left|\Psi_{0}\right\rangle=\left[\mathcal{g}_{0}(0, \mathcal{E})+\sum_{n=1}^{\infty} \frac{\delta^{n} \mathcal{g}_{0}(0, \mathcal{E})}{\delta \mathcal{E}^{n}}(\Delta E)^{n}\right]_{\mathcal{E}=E_{0}}\left|\Psi_{0}\right\rangle,
$$

where $\Delta E$ is the difference between the exact energy $E$ and the unperturbed energy $E_{0}$ and $V_{\text {eff }}\left|\Psi_{0}\right\rangle=\Delta E\left|\Psi_{0}\right\rangle$. This is a Taylor expansion, (In the limit of complete degeneracy we have (see further $\left[10\right.$, Appendix E]) $\delta^{n} / \delta \mathcal{E}^{n} \Rightarrow(1 / n !)$ $\left.\left(\partial^{n} / \partial \varepsilon^{n}\right)\right)$ and the result can be expressed

$$
g\left(0, E_{0}\right)\left|\Psi_{0}\right\rangle=g_{0}(0, E)\left|\Psi_{0}\right\rangle \text {, }
$$

implying that the MSC have the effect of shifting the energy parameters from the unperturbed to the perturbed energy. This relation is analogous to the relation between the Brillouin-Wigner and the Rayleigh-Schrödinger expansions in perturbation theory (see, for instance, [1, Chapter 9]).

It can be shown that analogous results hold also for a general model space and for arbitrary times. This implies that also the time dependence of the Green's operator is shifted in a similar way. In view of (33) the time dependence of the GO, operating on a model state $\Psi_{0}^{\alpha}$ then becomes $\mathrm{e}^{-\mathrm{i} t\left(E^{\alpha}-H_{0}\right)}$ (in interaction picture). Since according to (41) Green's operator has the same time dependence as the relativistic wave function, this result is in accordance with the standard quantum-mechanical picture. It then follows that

$$
\mathrm{i}\left(\frac{\partial}{\partial t} g(t,-\infty)\right)_{t=0}\left|\Psi_{0}^{\alpha}\right\rangle=\left(E^{\alpha}-H_{0}\right)\left|\Psi^{\alpha}\right\rangle .
$$

Projecting onto the model space, yields

$$
V_{\text {Feff }}=P\left(\mathrm{i} \frac{\partial}{\partial t} g(t,-\infty)\right)_{t=0} P
$$

using the definition (11).

3.5. Connection to the Bethe-Salpeter Equation. (See [10], [6, Section 6.9].) We shall now show that the procedure presented here is compatible with the Bethe-Salpeter equation $[24,25]$. We consider a single target state $|\Psi\rangle$ (with energy $E$ ) and the model state $\left|\Psi_{0}\right\rangle$ (energy $E_{0}$ ). From the relations (43) and (53) we have

$$
\begin{aligned}
\Omega & =g_{(}\left(0, E_{0}\right)=g_{0}(0, E) \\
& =1+\Gamma_{Q}(E) V(E)+\Gamma_{Q}(E) V(E) \Gamma_{Q}(E) V(E)+\cdots
\end{aligned}
$$

which is a Brillouin-Wigner expansion of the wave operator. We then have

$$
\begin{aligned}
Q(E & \left.-H_{0}\right) \Omega\left|\Psi_{0}\right\rangle \\
& =Q\left(V(E)+V(E) \Gamma_{Q}(E) V(E)+\cdots\right)\left|\Psi_{0}\right\rangle \\
& =Q V(E) \Omega\left|\Psi_{0}\right\rangle .
\end{aligned}
$$

In analogy with the relation (53) we have for the effective interaction (11)

$$
V_{\text {eff }}\left(E_{0}\right)=V_{\text {eff, } 0}(E)
$$

where

$$
\begin{aligned}
& V_{\text {eff, } 0}(E) \\
& \quad=P\left(V(E)+V(E) \Gamma_{Q}(E) V(E)+\cdots\right) P=P V(E) \Omega P
\end{aligned}
$$

is the effective interaction without folds. Hence,

$$
V_{\text {eff }}\left(E_{0}\right)\left|\Psi_{0}\right\rangle=P V(E) \Omega\left|\Psi_{0}\right\rangle
$$

But according to the definition (11)

$$
V_{\text {eff }}\left|\Psi_{0}\right\rangle=P\left(E-H_{0}\right)\left|\Psi_{0}\right\rangle=P\left(E-H_{0}\right) \Omega\left|\Psi_{0}\right\rangle,
$$

since $P \Omega P=P$ in intermediate normalization and $P$ commutes with $H_{0}$. Combining this with (57) leads to the relation

$$
\left(E-H_{0}\right) \Omega\left|\Psi_{0}\right\rangle=V(E) \Omega\left|\Psi_{0}\right\rangle .
$$

The treatment can be generalized by replacing the singlephoton exchange by the set of all irreducible multiphoton exchange, $\mathcal{V}$ (see Figure 8), leading to the "Schrödinger-like" equation in the restricted Hilbert space [10, Equation (113)], [6, Equation (6.126)]

$$
\left(H_{0}+\mathcal{V}(E)\right)|\Psi\rangle=E|\Psi\rangle
$$

which is equivalent to the Bethe-Salpeter equation $[24,25]$.

In the restricted space we have an equivalent Hamiltonian

$$
H=H_{0}+\mathcal{V}(E)
$$

that is energy dependent. This we can compare with the GellMann-Low Hamiltonian (44) that is energy independent and operates in the extended Fock space.

\section{Combining MBPT with QED}

4.1. General. (See [11], [6, Section 8.3].) We shall now describe how the covariant-evolution operator (CEO), developed in the previous section, can be used to combine MBPT and QED into a unified theory.

What is conventionally referred to as QED effects are effects that lie beyond the standard relativistic MBPT treatment, based upon the relativistic Dirac-Coulomb-Breit Hamiltonian (19). These effects are of three kinds, (i) retardation, (ii) virtual electron-positron pairs, and (iii) radiative effects (electron self-energy, vacuum polarization, and vertex correction).

The retardation effect is considered in the photon exchange above (30). Virtual pairs can be treated together with 


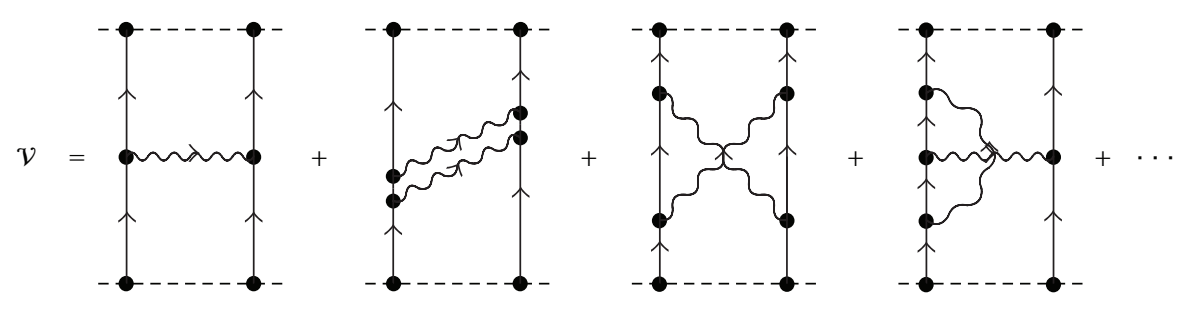

Figure 8: Examples of irreducible multiphoton exchange diagrams.
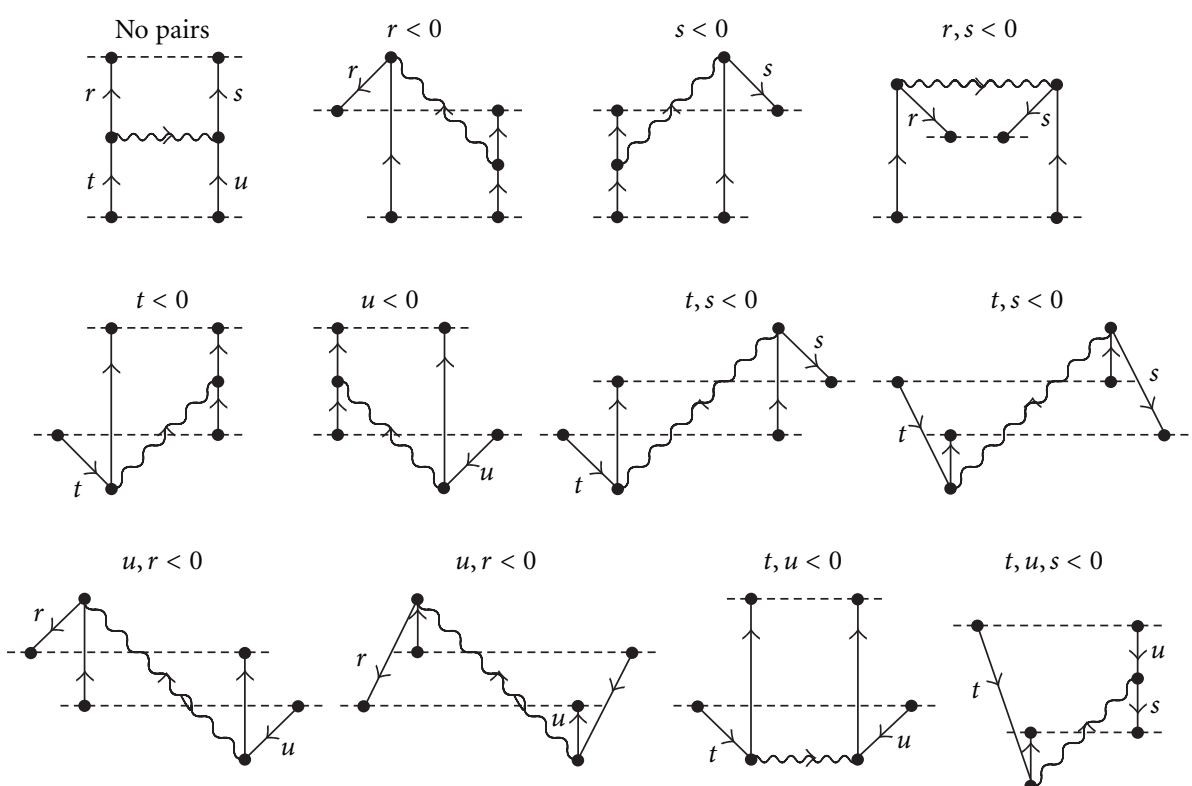

$t, u, s<0$
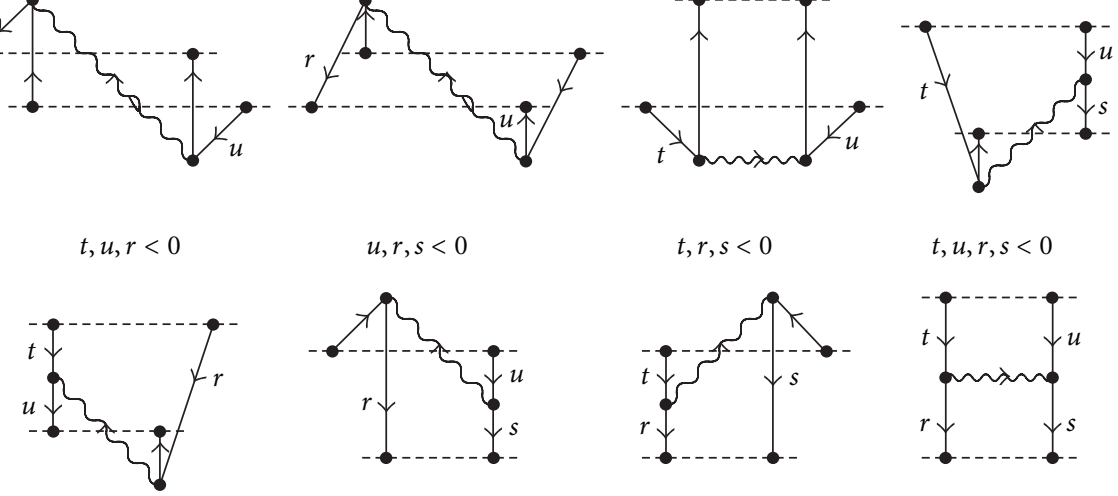

FIGURE 9: All 16 time-ordered diagrams corresponding to the transverse single-photon exchange given by $(65)$ ([6, Figure 8.3]).

single-photon exchange by generalizing the potential to $[6$, Equation (8.11)]

$$
\begin{aligned}
\left\langle r s\left|V_{\mathrm{sp}}^{\mathrm{VP}}(\mathcal{E})\right| t u\right\rangle & \\
=\langle r s| \int \mathrm{d} \kappa f(\kappa)[ & \pm \frac{t_{ \pm} r_{\mp}}{\varepsilon_{t}-\varepsilon_{r} \pm \kappa} \pm \frac{t_{ \pm} s_{ \pm}}{\mathcal{E}-\varepsilon_{t}-\varepsilon_{s} \mp \kappa} \\
& \left. \pm \frac{u_{ \pm} r_{ \pm}}{\mathcal{E}-\varepsilon_{r}-\varepsilon_{u} \mp \kappa} \pm \frac{u_{ \pm} s_{\mp}}{\varepsilon_{u}-\varepsilon_{s} \pm \kappa}\right]|t u\rangle,
\end{aligned}
$$

where $t_{ \pm}$, and so forth, represent projection operators for particle/hole states, respectively. The upper or lower sign should be used consistently in each term, inclusive the sign in the front. The 16 combinations of upper and lower signs in the four term lead to the time-ordered diagrams, shown in Figure 9.

Vacuum-polarization effects on electron propagators can be represented by a potential, as discussed in several publications [26].

The single-photon exchange is in the covariant-evolution operator procedure represented by two single-particle interactions (23). The first-order electron self-energy can then be evaluated by closing the second interaction on the same electron (c.f. Figure 4). In both cases-before closing the photon-one or several instantaneous interactions (Coulomb or Breit) could be inserted, leading to the "QED potential", illustrated in Figure 10. It is assumed here that the selfenergy and vertex diagrams are properly renormalized (see further below). This potential contains at most one retarded photon, which is the maximum that for the time being can 


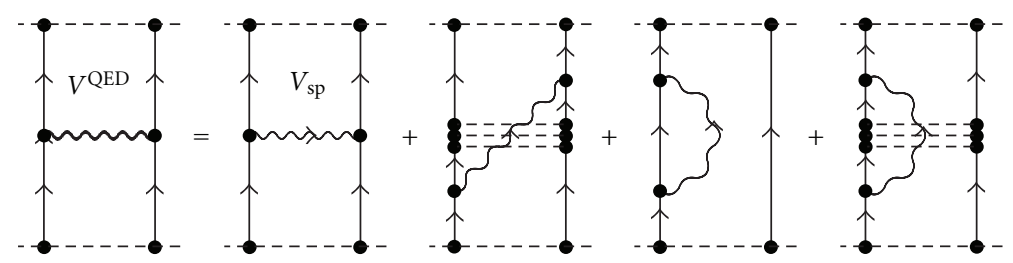

FIGURE 10: Feynman diagram representing the "QED potential," $V^{\mathrm{QED}}$. The dotted lines represent instantaneous Coulomb and (if needed) Breit interactions [6, Figure 8.10].
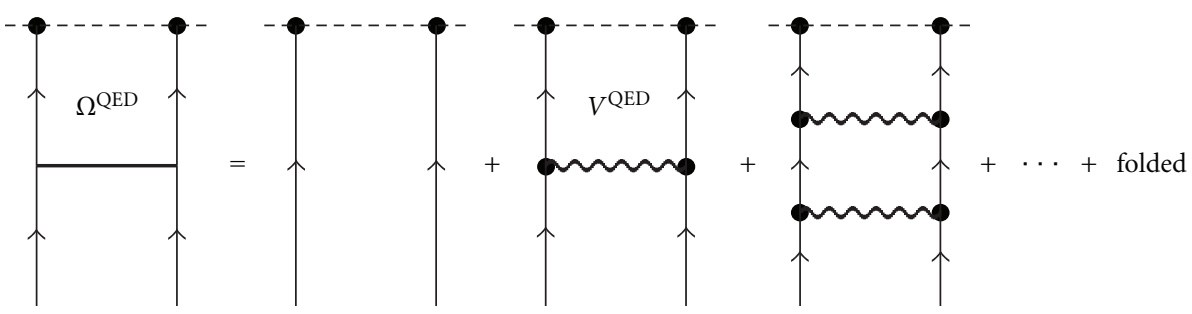

FIGURE 11: Iteration of the QED potential in Figure 10. This can be expressed by the self-consistent Dyson-type equation in Figure 12 [6, Figure 8.13].
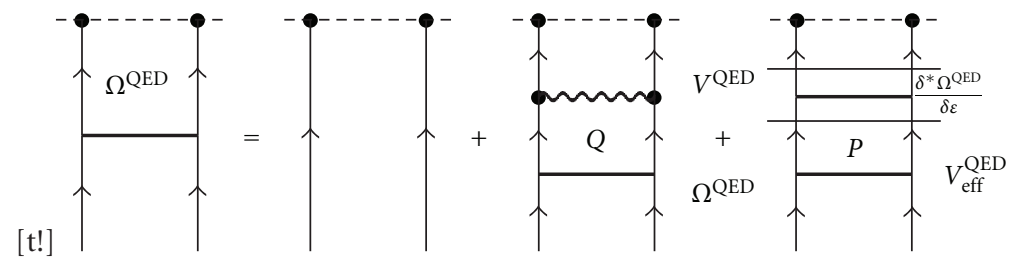

FIGURE 12: Graphical representation of the single-photon Bloch equation (66) (c.f. Figure 3). The last diagram represents the "folded" term, that is, the last term of the equation. This equation can be compared with the Bethe-Salpeter equation, valid only in the single-reference case, where there is no folded contribution. The order-by-order expansion of this equation is illustrated in Figure 11 [6, Figure 8.12].

be handled numerically. It should be noted that by including one or several instantaneous Breit interactions, most of the higher-order effects are actually included. This approximation is therefore quite accurate, as will be demonstrated below.

The QED potential can be iterated, as illustrated in Figure 11. The sum can be expressed in a Dyson-type of equation [6, Equation (8.68)]

$$
\Omega^{\mathrm{QED}}=1+\Gamma_{\mathrm{Q}} V^{\mathrm{QED}} \Omega^{\mathrm{QED}}+\frac{\delta^{*} \Omega^{\mathrm{QED}}}{\delta \mathcal{E}} V_{\mathrm{eff}}^{\mathrm{QED}}
$$

which is equivalent to a Bloch equation (10). Here, the last term represents the folded contribution. The asterisk indicates that the partial derivation should only include the last interaction. This equation can be generalized by replacing the single-photon potential by the irreducible multiphoton potential (Figure 8). The equation, illustrated in Figure 12, then represents a generalization of the Bethe-Salpeter equation, valid also for a multidimensional model space. Due to its resemblance with the standard Bloch equation of MBPT (10), it is referred to as the Bethe-Salpeter-Bloch equation.

The Bethe-Salpeter equation as it stands, however, is not suitable for numerical work, since already in second order (two-photon exchange) it is essentially beyond reach for computers of today. This problem can be circumvented by using the covariant-evolution operator with (to start with) one retarded photon and a number of instantaneous ones, which can easily be generated. We may then start with an iterated pair function, $\Omega_{\mathrm{I}}$ (13), and add a transverse photon, with or without crossing Coulomb interactions, and finish with new Coulomb iterations. This leads to the modified Bethe-Salpeter equation [11], [6, Equation (8.70)],

$$
\Omega^{\mathrm{QED}}=\Omega_{\mathrm{I}}+\Gamma_{\mathrm{Q}} V^{\mathrm{QED}} \Omega^{\mathrm{QED}}+\frac{\delta^{*} \Omega^{\mathrm{QED}}}{\delta \mathcal{E}} V_{\mathrm{eff}}^{\mathrm{QED}}
$$

illustrated in Figure 13. This procedure leads to much faster convergence and is already with a single retarded photon in most cases more accurate than the second-order BSE.

4.2. Coupled-Cluster-QED Procedure. The procedure described above can also be combined with the coupled-cluster approach, mentioned earlier (Section 2.2). This leads to a CCQED procedure, described in a recent publication [27]. It is also briefly described in the book [6, Section 8.4]. The pair functions of the classical procedure are then replaced by pair functions including QED effects, and the single-particle interactions will include electron self-energy and other single-particle effects. Since the classical and the QED procedures are completely compatible, the QED effects need to be included only in the components, where the effect is expected to significant. 


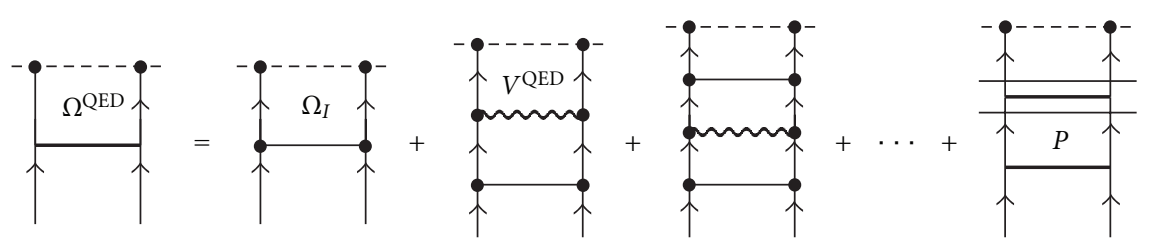

FIGURE 13: Graphical representation of the Bloch equation (67), where a standard pair function $\left(\Omega_{\mathrm{I}}\right)$ is combined with a QED potential [6, Figure 8.14].

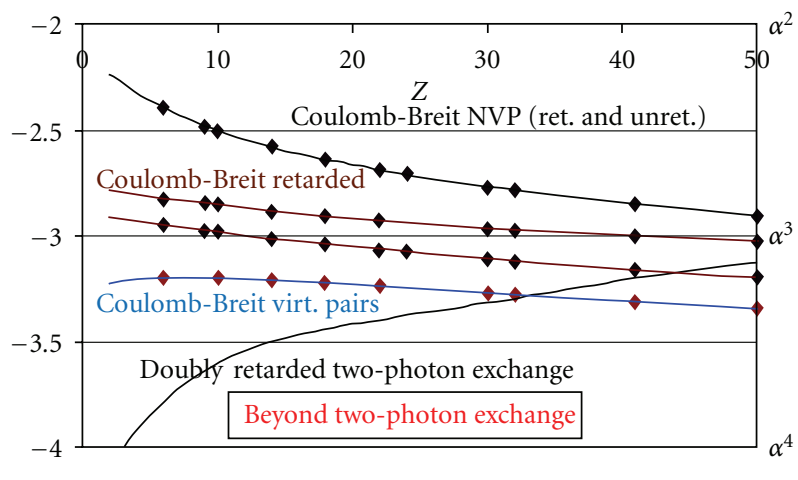

(a)
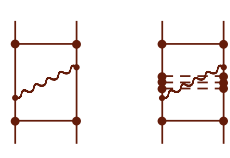

(b)
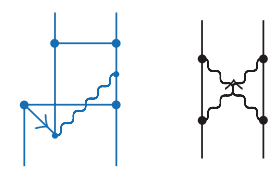

FIGURE 14: The effect of electron correlation beyond two-photon exchange-Coulomb-Breit NVPA, Coulomb-Breit retardation with and without Coulomb crossings, and Coulomb-Breit virtual pairs, all WITH electron correlation, for the ground-state of helium-like ions (from $[12,13])$. For comparison the effect of pure retarded two-photon exchange without additional correlation (included in the second-order Bethe-Salpeter equation) is also indicated [6, Figure 10.10].

4.3. Renormalization. (See [6, Chapter 12].) The radiative QED effects involve divergences that have to be eliminated by regularization and renormalization processes. In order to take full advantage of the developments in MBPT, it is necessary to perform the calculations using the Coulomb gauge. The renormalization process is more complicated in the non-covariant Coulomb gauge than in the covariant gauges normally used in QED calculations. However, formulas have been derived by Adkins for the dimensional regularization in Coulomb gauge for the lowest-order free-electron self-energy and vertex correction $[28,29]$. This procedure is now being implemented in our program structure $[14,15]$. It has been tested on the self-energy of highly charged hydrogenlike ions, and the results agree very well with those obtained earlier using Feynman gauge. In addition, the Coulombgauge results exhibit higher numerical stability. To our knowledge this represents the first numerical dimensional regularization in the Coulomb gauge.

\section{Implementation Procedure and Numerical Results}

We illustrate the results of the numerical implementation of the procedures briefly described in the previous sections by the calculations performed by Hedendahl in his doctorial thesis [12] and illustrated in Figure 14. The implementation procedure and some results are also summarized in [6, Chapter 10] (more details will be given in a forthcoming publication [13]).
The picture shows the effect of electron correlation beyond two-photon exchange for the Coulomb-Breit interaction on helium-like ions. The results are normalized to the nonrelativistic ionization energy, and the vertical scale is logarithmic. The top line represents the total correlation effect in the no-pair approximation. This effect is largely present in relativistic MBPT (19). The second and third lines show the effect of correlation upon the retardation, without and with Coulomb crossings (without virtual pairs), and the last heavy line represents the correlation effect on the virtual pairs. These effects lie beyond all previously existing procedures and, therefore, have never been calculated.

As a comparison, the thin line at the bottom indicates the estimated effect of two retarded interactions without correlation. This is included in the second-order Bethe-Salpeter equation while the other effects are not. This comparison demonstrates clearly that-for light and medium-heavy elements - even a single retarded photon together with instantaneous Coulomb interactions contains substantial effects not present in second-order BSE. The accuracy of the combined MBPT-QED procedure can be improved even further by including the instantaneous Breit interaction.

\section{Summary and Outlook}

The present article summarizes the research activity of the Gothenburg Atomic-Theory Group over the last decade. For the first time a unified theory of MBPT and QED has been developed and implemented on highly charged helium-like 
ions. The procedure is compatible with the Bethe-Salpeter equation (BSE), which is exact but intractable for practical work and leads already in second order to almost insurmountable problems. The unified MBPT-QED procedure is a way around this problem. Here, a limited number of retarded interactions are combined with numerous instantaneous interactions (Coulomb and Breit). It has been demonstrated that already a single retarded interaction together with instantaneous interactions leads for light and medium-heavy elements to much higher accuracy than second-order BSE (Figure 14). This implies that the combined MBPT-QED procedure is superior to a perturbation expansion of the original BSE. Therefore, much more accurate calculations can be performed than have so far been possible. This opens up possibilities of performing calculations that was previously inaccessible. Then comparison can be made with very accurate measurements of energy separations $[4,5]$, results that so far have not been explained. Obviously, the model proposed has implications also beyond the current project.

\section{Acknowledgments}

The author is very grateful to his close collaborator Johan Holmberg for excellent contributions to the joint work and for their permission to the author to publish this paper.

\section{References}

[1] I. Lindgren and J. Morrison, Atomic Many-Body Theory, Springer, Berlin, Germany, 2nd edition, 1986.

[2] P. ČCársky, J. Paldus, and J. Pittner, Recent Progress in Coupled Cluster Methods: Theory and Applications, Springer, New York, NY, USA, 2009.

[3] S. Fritzsche, P. Indelicato, and T. Stöhlker, "Relativistic quantum dynamics in strong fields: Photon emission from heavy, few-electron ions," Journal of Physics B: Atomic, Molecular and Optical Physics, vol. 38, no. 9, pp. S707-S726, 2005.

[4] E. G. Myers, H. S. Margolis, J. K. Thompson, M. A. Farmer, J. D. Silver, and M. R. Tarbutt, "Precision measurement of the 1s2p 3P2-3P1 fine structure interval in heliumlike fluorine," Physical Review Letters, vol. 82, no. 21, pp. 4200-4203, 1999.

[5] T. R. Devore, D. N. Crosby, and E. G. Myers, "Improved measurement of the $1 \mathrm{~s} 2 \mathrm{sS} 01-1 \mathrm{~s} 2 \mathrm{pP} 13$ interval in heliumlike silicon," Physical Review Letters, vol. 100, no. 24, Article ID 243001, 2008.

[6] I. Lindgren and A. M. Mårtensson, "Analysis of the atomic fine structure, using a nonrelativistic many-body and a relativistic central-field approach," Physical Review A, vol. 26, no. 6, pp. 3249-3267, 1982.

[7] P. M. W. Johnson and J. Sucher, Relativistic, Quantum Electrodynamic and Weak Interaction Effects in Atoms, vol. 189, American Institute of Physics, New York, NY, USA, 1989.

[8] I. Lindgren, B. Åsén, S. Salomonson, and A. M. MårtenssonPendrill, "QED procedure applied to the quasidegenerate finestructure levels of He-like ions," Physical Review A. Atomic, Molecular, and Optical Physics, vol. 64, no. 6, pp. 062505/1062505/5, 2001.

[9] I. Lindgren, S. Salomonson, and B. Åsén, "The covariantevolution-operator method in bound-state QED," Physics Reports, vol. 389, no. 4, pp. 161-261, 2004.
[10] I. Lindgren, S. Salomonson, and D. Hedendahl, "Many-bodyQED perturbation theory: Connection to the two-electron Bethe-Salpeter equation," Canadian Journal of Physics, vol. 83, no. 3, pp. 183-218, 2005.

[11] I. Lindgren, S. Salomonson, and D. Hedendahl, "Manybody procedure for energy-dependent perturbation: merging many-body perturbation theory with QED," Physical Review $A$, vol. 73, no. 6, 2006.

[12] D. Hedendahl, "Towards a Relativistic Covariant Many-Body Perturbation Theory," Ph.D. thesis, University of Gothenburg, Gothenburg, Sweden, 2010.

[13] D. Hedendahl, S. Salomonson, and I. Lindgren, Physical Review. In preparation.

[14] D. Hedendahl and J. Holmberg, "Coulomb-gauge self-energy calculation for high-Z hydrogen ions," accepted for publication in Physical Review A.

[15] J. Holmberg, "Scalar vertex operator for bound-state QED in the Coulomb gauge," Physical Review A, vol. 84, no. 6, Article ID 062504, 4 pages, 2011.

[16] I. Lindgren, "The Rayleigh-schrodinger perturbation and the linked-diagram theorem for a multi-configurational model space," Journal of Physics B, vol. 7, no. 18, pp. 2441-2470, 1974.

[17] K. A. Brueckner, "Many-body problem for strongly interacting particles. II. Linked cluster expansion," Physical Review, vol. 100, no. 1, pp. 36-45, 1955.

[18] J. Goldstone, "Derivation of the Brueckner many-body theory," Proceedings of the Royal Society of London Series A, vol. 239, no. 1217, pp. 267-279, 1957.

[19] B. H. Brandow, "Linked-cluster expansions for the nuclear many-body problem," Reviews of Modern Physics, vol. 39, no. 4, pp. 771-828, 1967.

[20] I. Lindgren, "A coupled-cluster approach to the many-body perturbation theory for open-shell systems," International Journal of Quantum Chemistry, vol. 14, supplement 12, pp. 33$58,1978$.

[21] J. Sucher, "Foundations of the relativistic theory of manyelectron atoms," Physical Review A, vol. 22, no. 2, pp. 348-362, 1980.

[22] G. E. Brown and D. G. Ravenhall, "On the interaction of two electrons," Proceedings of the Royal Society A, vol. 208, no. 1095, pp. 552-559, 1951.

[23] V. M. Shabaev, "Two-time Green's function method in quantum electrodynamics of high-Z few-electron atoms," Physics Reports, vol. 356, no. 3, pp. 119-228, 2002.

[24] M. Gell-Mann and F. Low, "Bound states in quantum field theory," Physical Review, vol. 84, no. 2, pp. 350-354, 1951.

[25] E. E. Salpeter and H. A. Bethe, "A relativistic equation for bound-state problems," Physical Review, vol. 84, no. 6, pp. 1232-1242, 1951.

[26] H. Persson, I. Lindgren, S. Salomonson, and P. Sunnergren, "Accurate vacuum-polarization calculations," Physical Review A, vol. 48, no. 4, pp. 2772-2778, 1993.

[27] I. Lindgren, S. Salomonson, and D. Hedendahl, "Coupled clusters and quantum electrodynamics," in Recent Progress in Coupled Cluster Methods: Theory and Applications, P. ČCársky, J. Paldus, and J. Pittner, Eds., pp. 357-374, Springer, New York, NY, USA, 2010.

[28] G. S. Adkins, "One-loop renormalization of coulomb-gauge QED,” Physical Review D, vol. 27, no. 8, pp. 1814-1820, 1983.

[29] G. S. Adkins, "One-loop vertex function in Coulomb-gauge QED,” Physical Review D, vol. 34, no. 8, pp. 2489-2492, 1986. 

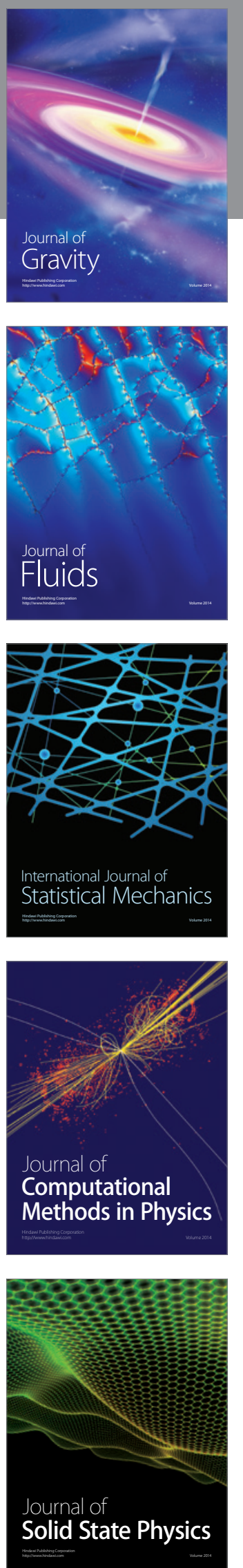

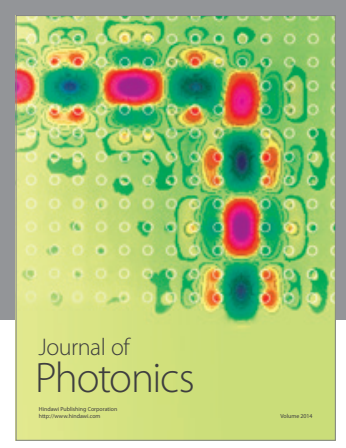

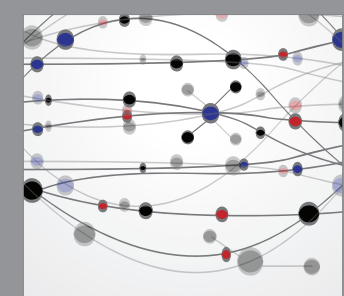

The Scientific World Journal
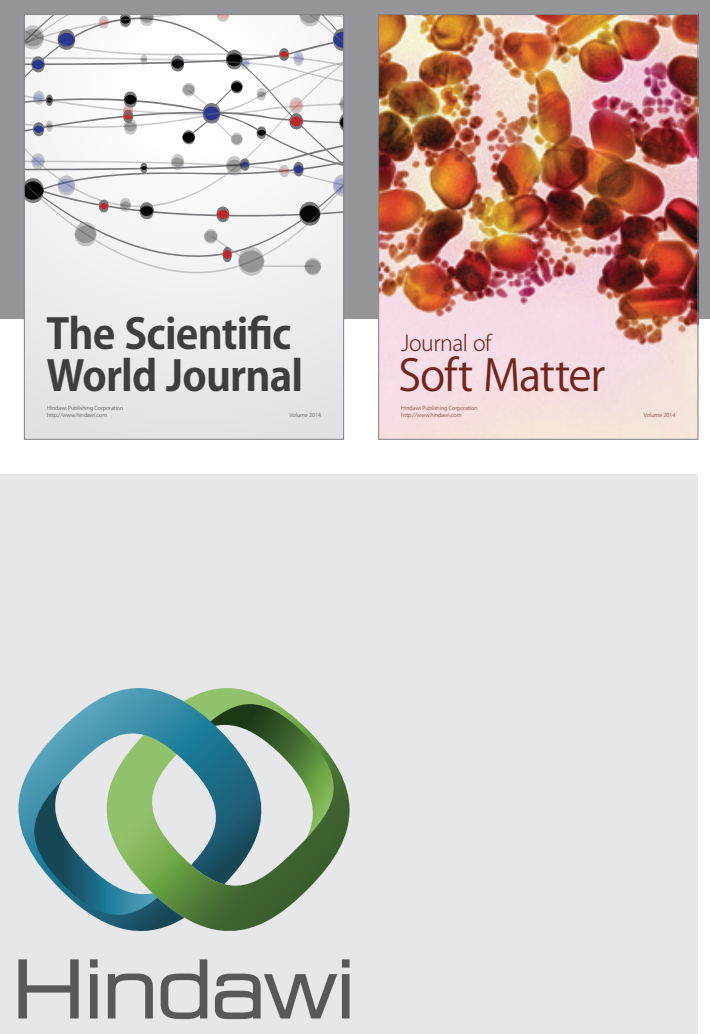

Submit your manuscripts at

http://www.hindawi.com
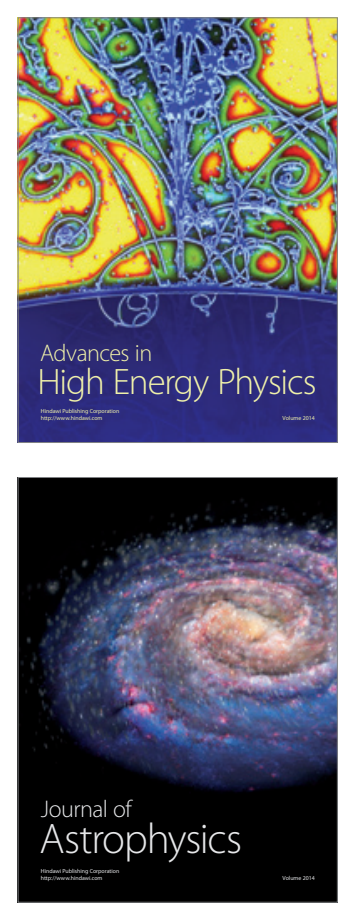
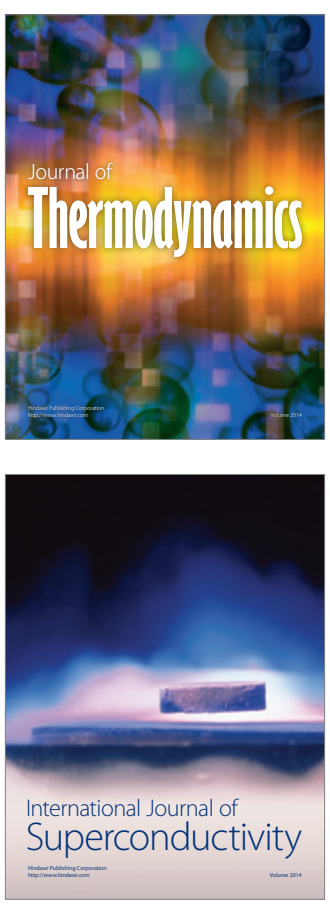
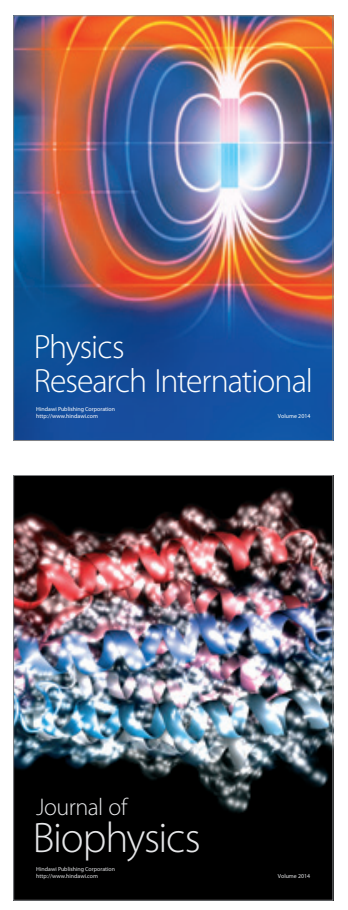
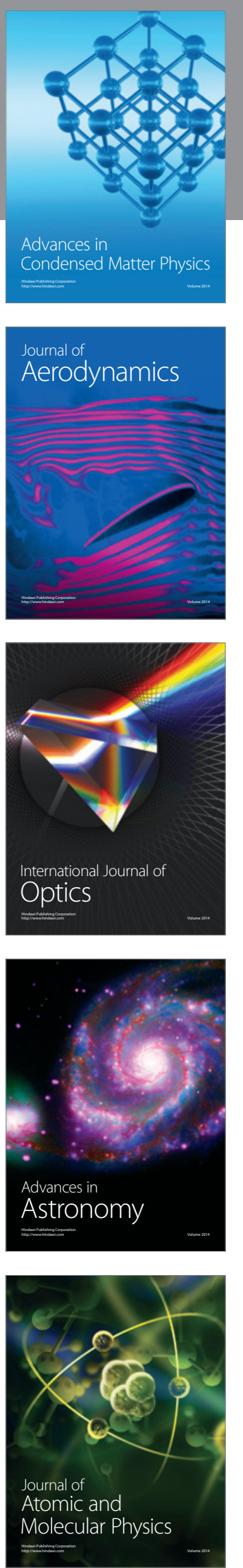